\title{
CYP3A5 Mediates Effects of Cocaine on Human Neocorticogenesis: Studies using an In Vitro 3D Self-Organized hPSC Model with a Single Cortex-Like Unit
}

\author{
Chun-Ting Lee*,1,2, Jia Chen', ${ }^{1,5}$ Abigail A Kindberg',5, Raphael M Bendriem', Charles E Spivak', \\ Melanie P Williams', Christopher T Richie', Annelie Handreck ${ }^{3}$, Barbara S Mallon ${ }^{4}$, Carl R Lupica', \\ Da-Ting Lin', Brandon K Harvey', Deborah C Mash² and William J Freed' \\ IIntramural Research Program (IRP), National Institute on Drug Abuse, National Institutes of Health (NIH), Baltimore, MD, USA; ${ }^{2}$ Department of \\ Neurology, Miller School of Medicine, University of Miami, Miami, FL, USA; ${ }^{3}$ Department of Pharmacology, Toxicology, and Pharmacy, University of \\ Veterinary Medicine, Hannover, Germany; ${ }^{4} \mathrm{NIH}$ Stem Cell Unit, IRP, National Institute of Neurological Disorders and Stroke, NIH, Bethesda, \\ MD, USA
}

\begin{abstract}
Because of unavoidable confounding variables in the direct study of human subjects, it has been difficult to unravel the effects of prenatal cocaine exposure on the human fetal brain, as well as the cellular and biochemical mechanisms involved. Here, we propose a novel approach using a human pluripotent stem cell (hPSC)-based 3D neocortical organoid model. This model retains essential features of human neocortical development by encompassing a single self-organized neocortical structure, without including an animal-derived gelatinous matrix. We reported previously that prenatal cocaine exposure to rats during the most active period of neural progenitor proliferation induces cytoarchitectural changes in the embryonic neocortex. We also identified a role of CYP450 and consequent oxidative ER stress signaling in these effects. However, because of differences between humans and rodents in neocorticogenesis and brain CYP metabolism, translation of the research findings from the rodent model to human brain development is uncertain. Using hPSC 3D neocortical organoids, we demonstrate that the effects of cocaine are mediated through CYP3A5-induced generation of reactive oxygen species, inhibition of neocortical progenitor cell proliferation, induction of premature neuronal differentiation, and interruption of neural tissue development. Furthermore, knockdown of CYP3A5 reversed these cocaine-induced pathological phenotypes, suggesting CYP3A5 as a therapeutic target to mitigate the deleterious neurodevelopmental effects of prenatal cocaine exposure in humans. Moreover, 3D organoid methodology provides an innovative platform for identifying adverse effects of abused psychostimulants and pharmaceutical agents, and can be adapted for use in neurodevelopmental disorders with genetic etiologies.

Neuropsychopharmacology (2017) 42, 774-784; doi:I0.I038/npp.2016.156; published online 2I September 2016
\end{abstract}

\section{INTRODUCTION}

It has been suggested that prenatal cocaine exposure impairs neurobehavioral development through disruption of frontal cortical development (Rando et al, 2013; Singer et al, 2002; Bandstra et al, 2004). However, elucidation of these effects in human subjects is fraught with difficulties related to numerous uncontrolled variables such as multidrug use, nutrient intake, and environmental factors (Bandstra et al, 2011; Singer et al, 2008). These potential confounds have made it challenging to define the adverse effects of prenatal cocaine exposure on human brain development, or to

*Correspondence: Dr C-T Lee, Center for Biologics Evaluation and Research, US Food and Drug Administration, Building 52, Room II21, 10903 New Hampshire Avenue, Silver Spring, MD 20993, USA, Tel: + 443895 80 I I,E-mail: Chun-Ting.Lee@fda.hhs.gov

${ }^{5}$ These two authors contributed equally to this work.

Received 10 March 2016; revised 26 May 2016; accepted 10 August 2016; accepted article preview online 18 August 2016 explore the mechanisms involved. Experiments in nonhuman primates reveal that prenatal cocaine exposure during the second trimester causes multiple neocortical cytoarchitectural abnormalities, including neuronal displacement in the cerebral wall, decreased numbers of cortical neurons, and decreased neocortical volume (Lidow et al, 2001; Lidow and Song, 2001). We previously demonstrated in rats that prenatal cocaine exposure during the most active period of neural progenitor proliferation induces cytoarchitectural changes in the embryonic neocortex (Lee et al, 2011). These cytoarchitectural changes are initiated by $\mathrm{N}$-oxidative metabolism of cocaine and consequent oxidative ER stress signaling (Lee et al, 2008). However, because of significant differences between humans and rodents in neocorticogenesis (Rakic, 2009; Hill and Walsh, 2005) and CYP-mediated drug metabolism (Martignoni et al, 2006), it has been difficult to translate findings from the rodent model directly to human brain development.

The specific CYP450 enzymes responsible for the oxidative metabolism of cocaine vary between species, such as CYP2A, 
$2 \mathrm{~B}$ and $3 \mathrm{~A}$ in mice, or $\mathrm{CYP} 2 \mathrm{~B}$ and $3 \mathrm{~A}$ in rats. In humans, only the CYP3A family metabolizes cocaine (Valente et al, 2012). There are four isoforms of human CYP3A: CYP 3A4, 3A5, 3A7, and 3A43. CYP3A4, the predominant isoform of CYP3A, and CYP3A5 are primarily expressed in the adult liver and small intestine. Nevertheless, CYP3A4 and CYP3A5 proteins and mRNA have been identified in the adult human brain, and their potential role in the metabolism of endoand xenobiotics in the brain have been emphasized (Booth Depaz et al, 2013; McFadyen et al, 1998). CYP3A7, primarily seen in the fetal liver, is sometimes expressed in the mature liver in small amounts. Finally, CYP3A43 is expressed in small amounts in the liver, but is expressed at higher levels in the brain. Because of low levels of expression in the liver, CYP3A43 has not been thought to play a critical role in drug metabolism; however, CYP3A43 may play a role in the metabolism of drugs in the human brain (Agarwal et al, 2008).

We recently described a two-dimensional (2D) in vitro model of human neocortical development using human pluripotent stem cells (hPSCs), and used this model to examine the effects of cocaine during neocorticogenesis (Kindberg et al, 2014). This 2D cell culture model does not, however, recapitulate in vivo cortical layer formation and cytoarchitectural organization, limiting its use for the understanding of cocaine-induced changes of brain morphogenesis, cellular function, and gene expression. Several three-dimensional (3D) in vitro models of the brain that could bridge the gap between 2D models and the normal brain in situ have been reported. Among these, a 3D cerebral organoid in vitro model system enables mechanistic studies of neocorticogenesis (Lancaster et al, 2013), perhaps providing a new model for drug discovery. However, the use of Matrigel embedding introduces undefined animal factors, and interferes with penetration of drugs and other compounds into the organoids. Therefore, this method would not be suitable for some pharmacological applications. Another $3 \mathrm{D}$ culture system of human cortical spheroids (hCSs) allows for examination of human cortical development, without embedding into a gelatinous scaffold (Paşca et al, 2015). This $3 \mathrm{D}$ cell culture model, however, employs multiple, and varying, numbers of ventricular zone (VZ)-like regions within each hCS that can result in difficulties in reproducibility and quantification of the cytoarchitecture.

Here we describe an hPSC-based 3D neocortical organoid model that develops as a single self-organized structure, using defined patterning molecules and neocortical trophic factors without extracellular scaffolding. We determined that the size of hPSC-derived neuroepithelial (NE) rosettes, which are manually dissected for use in subsequent neocortical organoid differentiation, is critical for achieving sufficient neocortical organoid development, with small rosettes being unable to successfully differentiate. This 3D neocortical organoid model retains essential features of human neocortical development, including a proliferative neuroepithelium during the earlier development stage, neurogenesis, neuronal migration, and later enlargement of the neocortical area. We employed these 3D cultures to model cocaine-induced alterations in organization and patterning of neocortical development, and to determine the role of CYP3A5 in these alterations.

\section{MATERIALS AND METHODS}

\section{hPSC Culture}

The hESC lines H1 (P46-50), H9 (P49-61), and H14 (P5260), provided by WiCell Research Institute, CT2 (P91-98), provided by University of Connecticut Stem Cell Core, and hiPSC lines NIH-i5 (P33-35) and NIH-i7 (P34-38), provided by NIH Stem Cell Unit, were employed for the $3 \mathrm{D}$ neocortical organoid differentiation protocol. NIH-i5 and i7 were derived by retroviral transduction of individual human fibroblasts from two different donors (Mallon et al, 2013). No interbatch differences in the hPSC-derived neocortical organoids were observed. The hPSCs were propagated in feeder-dependent culture using irradiated mouse embryonic fibroblasts (Global Stem) (Lee et al, 2015a, b). For more details, see the Supplementary Materials and Methods.

\section{D Neocortical Organoid Differentiation}

The hPSCs were differentiated to neocortical organoids as shown schematically in Figure 1a. Undifferentiated hPSC colonies were picked up from feeder-dependent culture using $1 \mathrm{mg} / \mathrm{ml}$ Collagenase Type IV for $30 \mathrm{~min}$. The hPSC aggregates were preserved, allowing embryoid body (EB) formation (day 0). EBs were grown, floating, in hPSC medium (see the Supplementary Materials and Methods) without bFGF but including $10 \mu \mathrm{M}$ SB431542 (Tocris), $0.1 \mu \mathrm{M}$ LDN193189 (Stemgent), and $1 \mu \mathrm{M}$ PD0325901 (Axon Medchem) for 4 days. The EBs were then transferred to neural media containing DMEM/F-12 (2:1) with N2 supplement, $0.1 \mathrm{mM}$ non-essential amino acids, and $2 \mu \mathrm{g} /$ $\mathrm{ml}$ heparin, supplemented with $20 \mathrm{ng} / \mathrm{ml}$ bFGF from days 4 to 6 . Colonies were then grown in adherent culture, on laminin-coated plates, in the same media from days 6 to 16 . On day 16, dorsal cortical rosettes ranging from 250 to $500 \mu \mathrm{m}$ in diameter $(\sim 50000$ to $200000 \mu \mathrm{m} 2)$ were isolated by manual dissection and primed in suspended culture with $20 \mathrm{ng} / \mathrm{ml} \mathrm{bFGF}$ and $20 \mathrm{ng} / \mathrm{ml}$ FGF18 for 8 days before being switched to neuronal differentiation media, containing neurobasal medium, B27 supplement, $0.1 \mathrm{mM}$ non-essential amino acids, $0.5 \mathrm{mM}$ L-glutamine, and $2 \mu \mathrm{g} / \mathrm{ml}$ heparin for 6 weeks in suspension conditions to generate self-organized neocortical organoids.

\section{Immunocytochemistry, Cell Counting, and TUJ1 ${ }^{+}$ Neocortical Area Analysis}

Adherent cells were fixed with $4 \%$ paraformaldehyde for $10 \mathrm{~min}$ and washed with PBS. Neocortical organoids were embedded in collagen gel, immersed in $4 \%$ paraformaldehyde for $20 \mathrm{~min}$, and cryoprotected in $20 \%$ sucrose in PBS for $20 \mathrm{~min}$.

Cryostat sections $(10 \mu \mathrm{m})$ were thaw-mounted onto gelatin-subbed slides. For both cells and tissue sections, $0.2 \%$ Triton X-100 in PBS containing 5\% BSA and 10\% goat serum was used as a blocking reagent. Cells were then incubated with primary antibodies (Supplementary Table S1) in PBS containing $0.2 \%$ Triton X-100, 5\% BSA, and 5\% goat serum. Fluorescent-labeled secondary antibodies Alexa-Fluor 488 (green) or Alexa-Fluor 555 (red; R\&D Systems) appropriate for each respective primary antibody were used. 


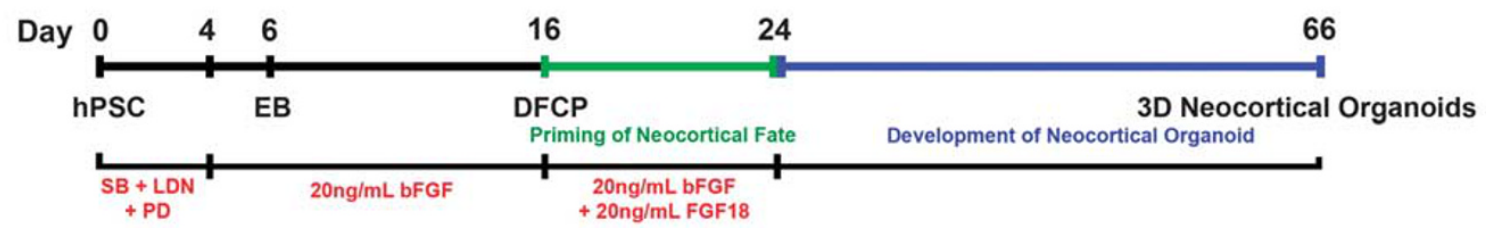

b
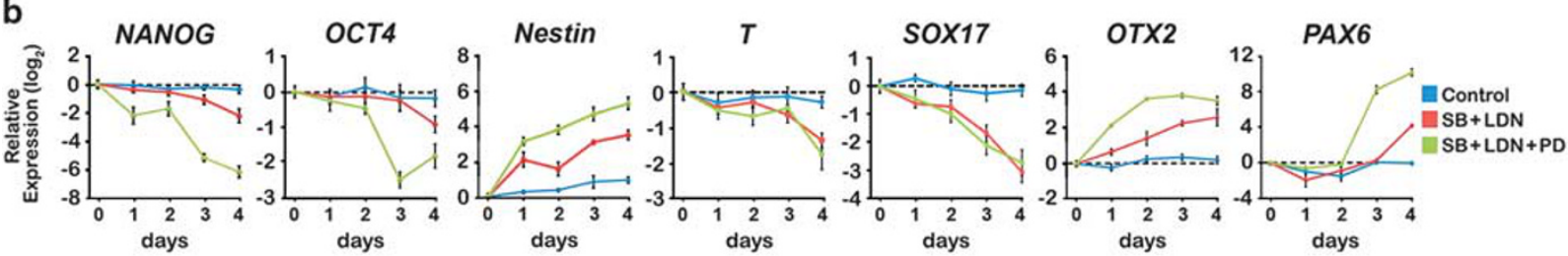

C

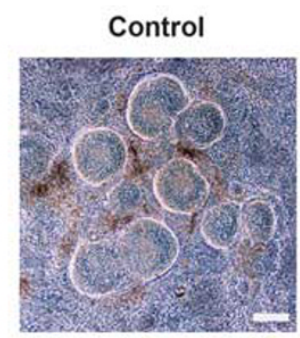

SB+LDN SB+LDN+PD
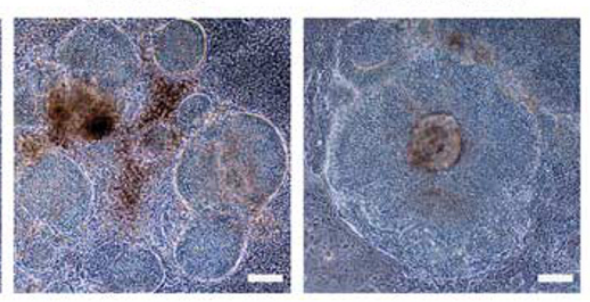

d

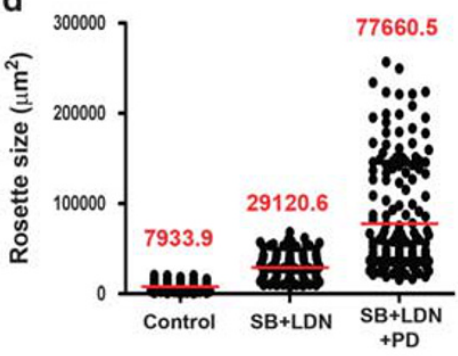

e

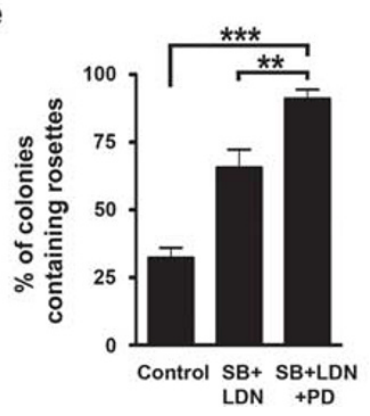

f Nestin HOXB4
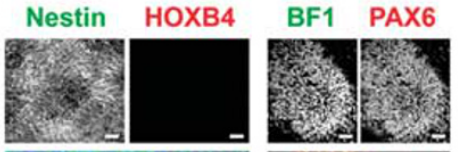

EMX1 NKX2.1
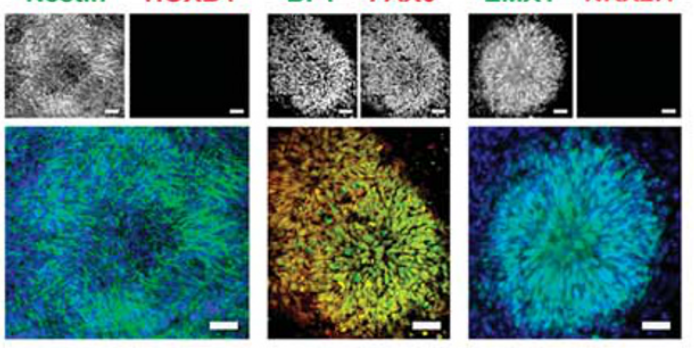

Figure I Combined dual SMAD and FGF inhibition induces efficient neocortical neuroectodermal conversion of hPSCs. (a) Schematic representation of the human neocortical organoid differentiation procedure. hPSC: human pluripotent stem cells; EB: embryoid bodies; DFCP: dorsal forebrain cortical

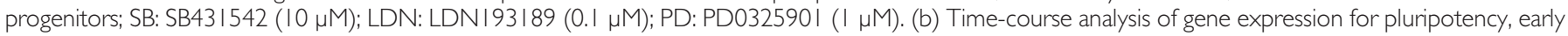
germ layer, and neural markers; $n=3$. (c) Phase-contrast images of NE rosettes derived from hPSCs at day I6. Scale bar, I00 $\mu m$. (d) Distribution of sizes of rosettes at day 16;n=128 for control, 142 for SB+LDN, and 176 for SB+LDN+PD, from three independent experiments. Red line indicates mean. (e) Analysis of percentages of colonies containing rosettes at day 16; $n=9$ independent experiments; total counted colonies: 506 for control, 573 for SB $+\mathrm{LDN}$, and 490 for SB+LDN+PD. One-way ANOVA followed by Tukey's compromise post hoc test; **P $<0.01$ and **** $<<0.001$. (f) Neocortical NE generated from hPSCs. Expression of Nestin/HOXB4, BFI/PAX6, and EMXI/ NKX2.I by immunocytochemistry at day I6. Scale bar, $50 \mu \mathrm{m}$. Data are shown as means \pm SEM in (b) and (e). Cell line: $\mathrm{H} 9$.

A Carl Zeiss Axiovert 200M microscope was used to capture the images. Cell death in the neocortical organoids was determined using the TUNEL In Situ Cell Death Detection Kit, Fluorescein (Roche) according to the manufacturer's instructions. For immunostaining of glutamate, VGLUT1, VGLUT2, GABA, and VGAT (Figure $2 \mathrm{~m}-\mathrm{q}$ ), neocortical organoids were gently mechanically disrupted by pipetting and were then seeded onto poly-ornithine/lamanin-coated plates overnight before staining. For quantification of BrdUlabeled $\mathrm{PAX6}^{+}$cellular percentages and percentages of $\mathrm{BrdU}^{+}$ cells expressing TUJ1, cells in three randomly selected fields each containing 50 to 150 cells were counted for 5 to 7 independent neocortical organoid samples. For $\mathrm{TUJ1}^{+}$ neocortical area analysis, organoids were cultured in individual wells of 96-well plates at day 24 . TUJ1 ${ }^{+}$neocortical areas were calculated by normalization of $\mathrm{TUJ} 1^{+}$areas in the neocortical organoids at day 66 (using only sections from the center of each organoid) to overall organoid size at day 24 using NIH ImageJ. For quantification of percentages of $\mathrm{TUJ}^{+}$cells expressing glutamate or GABA and GFAP ${ }^{+}$cells, neocortical organoids were dissociated into single cells using acutase before being reseeded onto poly-ornithine/lamanincoated plates for quantification. Cells in three randomly selected fields each containing 53 to 143 cells were counted for five independent cultures.

\section{Statistical Analysis}

Statistical analyses were performed with GraphPad InStat. Data are shown as means \pm SEM. Testing for normality was 
performed using the Kolmogorov-Smirnov test. For twogroup comparisons, unpaired two-tailed Student's $t$-tests were used for normally distributed data. For multiple comparisons, normally distributed data were analyzed by one-way ANOVA followed by Tukey's compromise post hoc test. Statistical significance of data with nonnormal distribution was analyzed using Mann-Whitney U-tests for two groups, or for more than two groups Dunn's multiple comparisons test was used. The criterion for statistical significance was $P<0.05$.

\section{RESULTS}

\section{Generation of Appropriate Size of Dorsopallial NE Rosettes for Neocortical Organoid Development from hPSCs by Combined Dual SMAD and FGF Inhibition}

Inhibition of dual SMAD signaling has been shown to dramatically enhance neural conversion of hPSCs (Chambers et al, 2009). In addition, FGF signaling at an early stage of hPSC differentiation has been shown to block neural differentiation (Greber et al, 2011). Here we tested whether combined blockade of both dual SMAD signaling, using LDN193189 and SB431542, and FGF signaling, using PD0325901, during EB formation, at the beginning of differentiation (days $0-4$ ), would achieve efficient neuroectoderm conversion from EBs, and generate NE rosettes of appropriate size for neocortical organoid differentiation (Figure 1a). We found that combined treatment with dual SMAD and FGF inhibitors accelerated the loss of pluripotency, indicated by rapid downregulation of NANOG and OCT4, and increased the efficacy of neuroectodermal differentiation indicated by upregulation of Nestin (Figure 1b). In addition, blocking both dual SMAD and FGF signaling suppressed mesodermal and endodermal marker expression, as indicated by downregulation of $T$ and SOX17, but this effect was replicated by dual SMAD blockade alone (Figure 1b).

The effect of combined dual SMAD and FGF inhibition on departure from self-renewal is presumably related to destabilization of the TGF $\beta /$ activin and FGF-regulated NANOG and OCT4 transcription network (Chambers et al, 2009; Greber et al, 2011). Because mesodermal and endodermal lineages are suppressed by dual SMAD inhibition (Figure 1b), suppression of TGF $\beta$ /activin and FGFregulated pluripotency enhances differentiation of hPSCs toward the neuroectodermal lineage (Figure 1b). It has been suggested that FGF/ERK inactivation increases expression of OTX2, an anterior telencephalic transcription factor, in hPSCs (Greber et al, 2011). Here, we also demonstrated that inhibition of dual SMAD and FGF signaling rapidly induced expression of OTX2 before the expression of the dorsal forebrain marker PAX6 (Figure 1b). Moreover, PAX6 induction started at day 3, when NANOG and OCT4 were almost maximally downregulated (Figure 1b). These results coincide with previous findings that OTX2 acts as a positive regulator of PAX6, whereas NANOG and OCT4 act as negative regulators (Greber et al, 2011). In addition, blockade of dual SMAD and FGF signaling did not change expression of alternative brain region markers, such as the hindbrain/ spinal cord marker $H O X B 4$, ventral telencephalic marker NKX2.1, and midbrain marker LMX1A (Supplementary
Figure S1a). Taken together, these data indicate that LDN193189, SB431542, and PD0325901 work synergistically to achieve efficient neocortical neuroectodermal conversion of hPSCs.

The neocortical trophic factor, bFGF, known to play a role in establishing the dorsal NE of the anterior neocortex (Raballo et al, 2000), was used to supplement conversion from EB to NE cells from days 4 to 16 (Figure 1a). The effects of inhibiting the dual SMAD and FGF pathways during the first 4 days of differentiation, in the context of subsequent supplementation with bFGF, on NE rosettes was characterized at day 16. A significant increase in rosette size was observed upon treatment with LDN193189, SB431542, and PD0325901 (a 9.8-fold increase $v s$ control; a 2.7-fold increase vs LDN193189 and SB431542) (Figure 1c and d). Later experiments showed that a rosette size of $50000 \mu \mathrm{m}^{2}$ or larger was critical for neocortical organoid growth with characteristic cerebral cortical organization (Supplementary Figure S3). Moreover, this protocol significantly increased the percentage of colonies containing NE rosettes, facilitating the collecting of rosettes for subsequent organoid differentiation (Figure 1e). Importantly, nestin ${ }^{+} \mathrm{NE}$ rosettes with dual SMAD and FGF pathway inhibition expressed the anterior telencephalic transcription factor BF1, and the dorsal forebrain markers PAX6 and EMX1 (Figure 1f). Conversely, these rosettes did not express the hindbrain/spinal cord marker HOXB4 or the ventral telencephalic marker NKX2.1 (Figure 1f). Together, this indicates that LDN193189, SB431542, and PD0325901 treatment during the EB stage of differentiation, followed by supplementation with bFGF, efficiently yielded rosettes of appropriate size and of dorsopallial identity.

\section{Characterization of hPSC-Based 3D Neocortical Organoids}

On day 16, dorsopallial NE rosettes ranging from 250 to $500 \mu \mathrm{m}$ in diameter $\left(\sim 50000\right.$ to $\left.200000 \mu \mathrm{m}^{2}\right)$ were manually picked up, maintained in suspension culture, and primed with bFGF, which has been shown to maintain the proliferative NE pool (Raballo et al, 2000), and FGF18, which is involved in neocortical patterning and development (Hasegawa et al, 2004), for 8 days. All trophic factors were then removed from the cultures during the final 6 weeks of differentiation to allow self-organized neocortical organoid formation (Figure 1a). Early-stage neocortical organoids (day 38) included large, proliferating polarized neuroepithelia, with single ventricle-like cavities at the centers (Figure $2 \mathrm{a}-\mathrm{d}$ and Supplementary Figure S4a and b). Dorsopallial progenitors in the VZ-like zones expressed BF1 and PAX6 and were proliferative, as they expressed Ki67, a cell proliferation marker (Figure 2b). The apical-end markers, N-cadherin (Ncad), CD133, and gamma-tubulin (g-tubulin) were localized to the center of each organoid, facing the lumen (Figure 2c). Moreover, PH3 (phosphorylated histone H3), a marker for the G2/M phase of the cell cycle, was detected in the apical zone of the neocortical organoids (Figure 2d) that was reminiscent of in vivo interkinetic nuclear movement in the VZ of the developing neocortex (Takahashi et al, 1993). On day 38, the neuronal marker TUJ1 was located superficial and peripheral to the $\mathrm{PAX}^{+}$neuroepithelium in the neocortical organoids (Figure 2e), and early-born preplate 
$\mathrm{TBR}^{+}$neurons were also located in areas surrounding the neuroepithelia (Figure 2f). REELIN ${ }^{+}$cells were found in the most superficial layer of the $\mathrm{TBR}^{+}$zone (Figure $2 \mathrm{~g}$ ) that is likely indicative of Cajal-Retzius cells that are essential for the construction of the cortical plate (Supèr and Uylings, 2001). By the end of differentiation on day 66, the average size of organoids and $\mathrm{TUJ1}^{+}$area became larger (Figure $2 \mathrm{~h}$ and i; Supplementary Figure S4d), and both early cortical
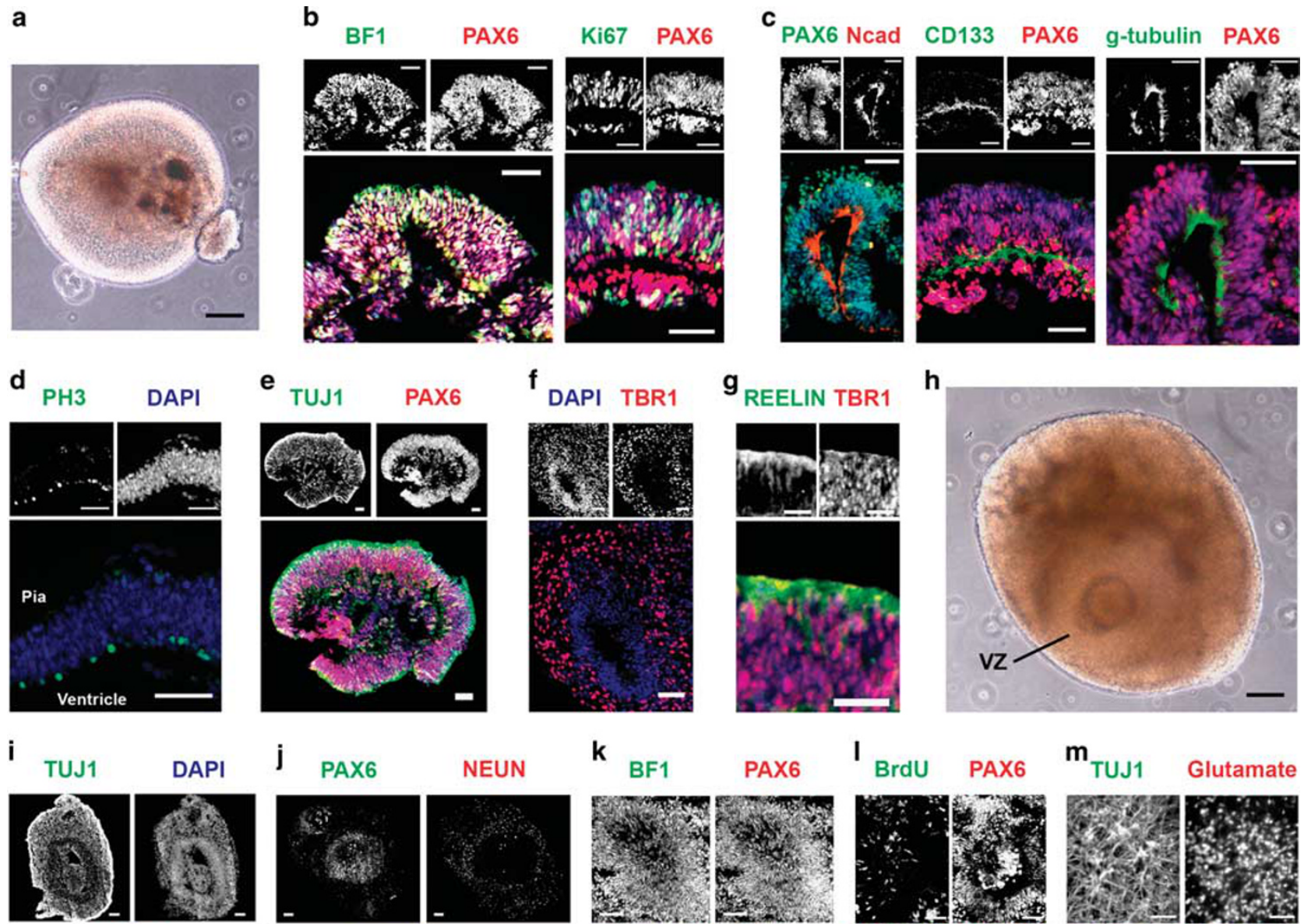

PAX6

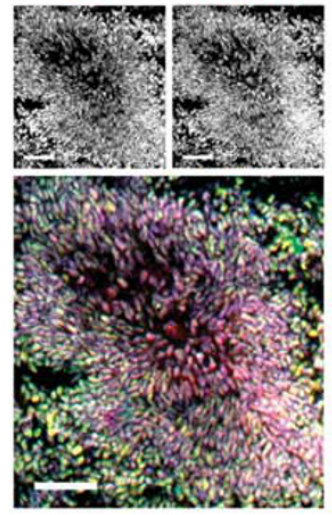

I Brdu

PAX6
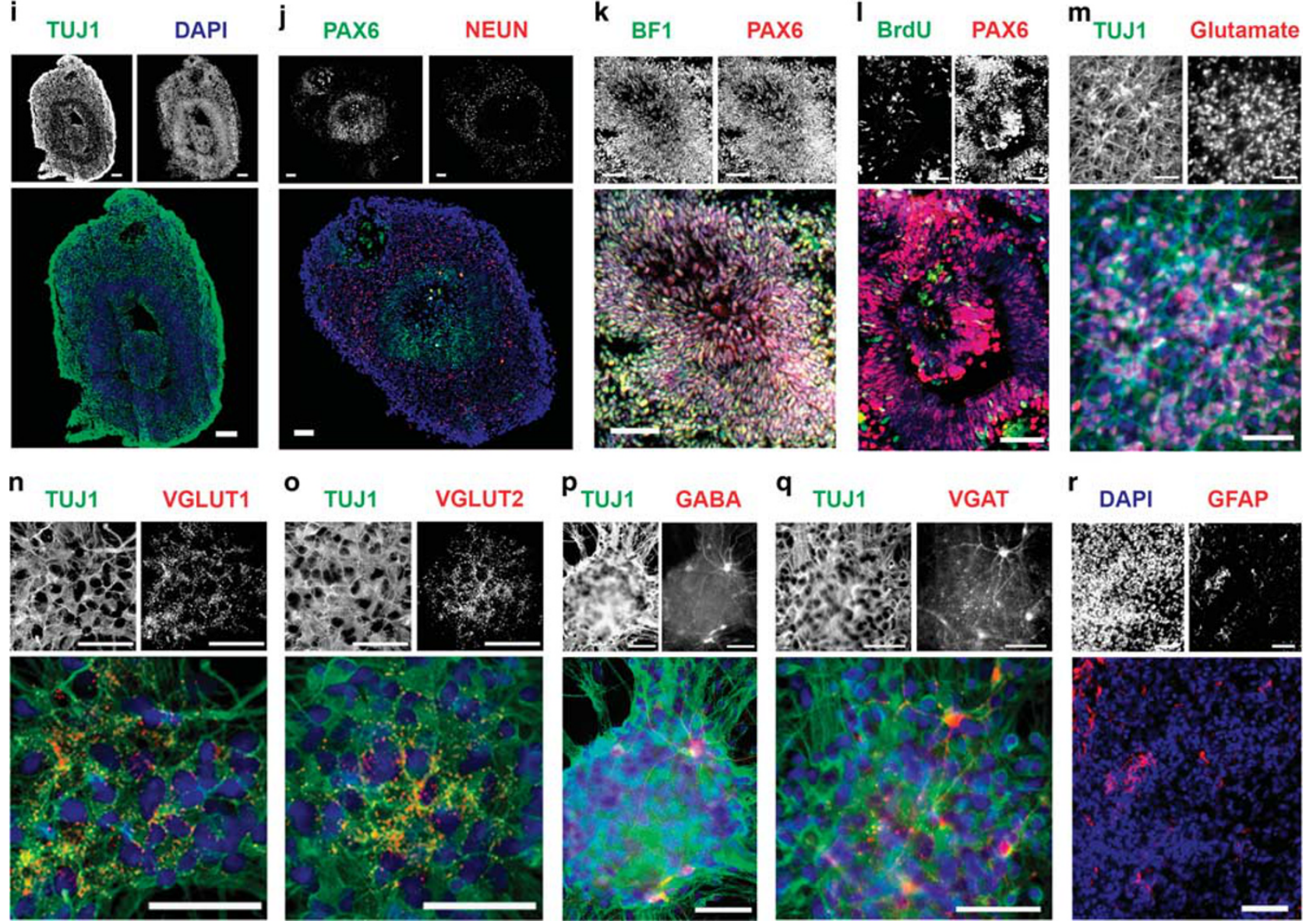
plate $\mathrm{CTIP}_{2}{ }^{+}$neurons and late cortical plate $\mathrm{CUX1}^{+}$neurons were present (Supplementary Figure S1b). The CTIP2 ${ }^{+}$ neurons were primarily located adjacent to the neuroepithelia; however, the $\mathrm{CUX1}^{+}$neurons were located evenly throughout the $\mathrm{TUJ1}^{+}$area (Supplementary Figure S1b), suggesting initiation of spatial separation of early and late cortical plate neurons. In addition, the neuronal maturation maker NEUN, which is expressed in developing human fetal brain by the middle of the second trimester (Sarnat et al, 1998), was expressed peripheral to the neuroepithelia (Figure $2 \mathrm{j}$ and $\mathrm{k})$.

The proliferation rate of $\mathrm{PAX}^{+}$neuroepithelial cells was significantly lower on day 66 than on day 45 (Figure 21 for day 66 and Figure $4 \mathrm{~d}$ for day $45 ; 6.8 \% \pm 1.0$ SEM on day $66 v s$ $17.5 \% \pm 1.8$ SEM on day $45 ; n=5 ; P<0.001$; two-tailed Student's $t$-tests), coinciding with the increase in cell-cycle length of progenitor cells that undergo differentiative divisions in the VZ at later stages of cortical neurogenesis in vivo (Dehay and Kennedy, 2007). Cell death was observed in a minority of cells near the middle of each neocortical organoid that could be because of limited oxygen supply in the organoid cores (Supplementary Figure S1c). The majority of differentiated neurons in the neocortical organoids were glutamatergic $(73.1 \% \pm 4.0 \mathrm{SEM} ; n=5$; Figure $2 \mathrm{~m})$, expressing VGLUT1 and VGLUT2 (Figure $2 \mathrm{n}$ and $\mathrm{o}$ ). Only a small fraction of differentiated neurons were GABAergic $(4.6 \% \pm 0.8 \mathrm{SEM} ; n=5)$ (Figure $2 \mathrm{p}$ ), expressing VGAT (Figure 2q). Similar glutamatergic and GABAergic neuron subtype compositions have been found in the human neocortex (Rakic, 2009). At the end of differentiation on day 66 , we observed $5.7 \% \pm 0.8$ SEM $(n=5)$ GFAP $^{+}$ astrocytes in the neocortical organoids (Figure 2r). This coincides with the phase at which astrogenesis is initiated during the later phases of neocorticogenesis in vivo (Cameron and Rakic, 1991; Levison and Goldman, 1993). Intact-organoid immunohistochemistry using the newly developed CLARITY method (Chung et al, 2013) allowed antibody, in this case TUJ1, to label throughout the intact neocortical organoid, providing an alternative method for analysis of organoid cytoarchitecture (Supplementary Figure S2a-e).

Whole-cell electrophysiological recording was used to evaluate functional activity of the differentiated neocortical neurons. At day 66, cells under current clamp exhibited robust action potentials (Figure $3 \mathrm{a}-\mathrm{c}$ ). Large voltage-gated sodium currents and delayed rectifier potassium currents a
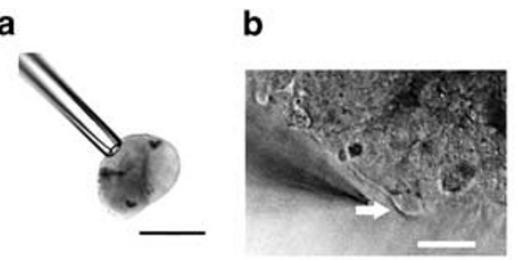

d
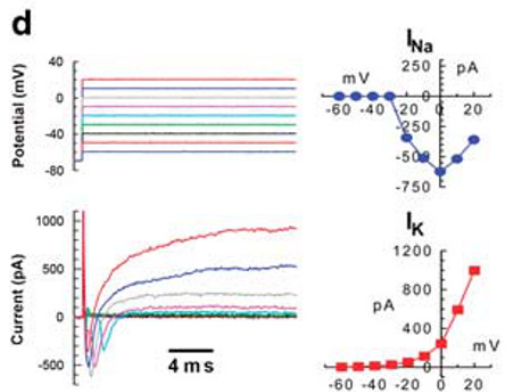

C

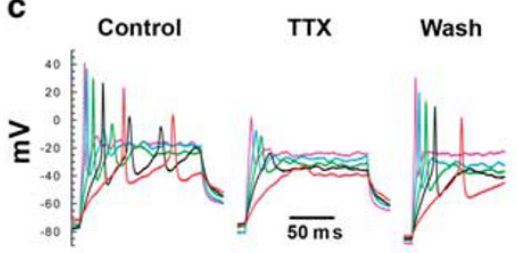

e

f
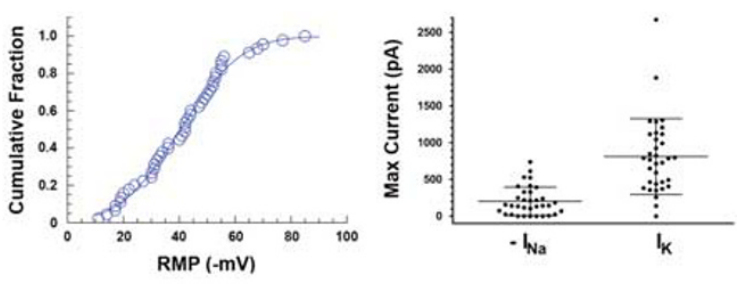

Figure 3 Electrophysiological analysis of neocortical neurons generated from hPSCs at day 66 by inhibition of dual SMAD and FGF signaling. (a) Typical neocortical organoid held with minimal suction by a suction pipette. Scale bar, $300 \mu \mathrm{m}$. (b) A patch pipette approaches a cell (indicated by arrow) on the organoid surface. Scale bar, $25 \mu \mathrm{m}$. (c) Voltage traces recorded in current clamp mode while injecting current in increasing increments. The strong action potentials recorded under control condition were blocked by tetrodotoxin, and recovered upon washing. (d) Voltage clamp recording from a cell with RMP of $-53 \mathrm{mV}$. Voltage commands (upper left) and corresponding current traces (lower left) are shown with the peak sodium (upper right) and potassium (lower right) currents plotted against membrane potential. (e) Cumulative distribution of resting membrane potentials $(n=45)$ follow a Gaussian distribution with a mean of $-40 \pm 17$ (SD) $\mathrm{mV}$. (f) Distributions of the peak sodium (left) and potassium currents (right). Each symbol represents a different cell. Means and SD are shown by horizontal bars. Cell line: H9.

Figure 2 Generation of 3D neocortical organoids with single ventricle-like cavities from hPSCs by combined dual SMAD and FGF inhibition. (a) Phasecontrast image of a floating neocortical organoid derived from hPSCs at day 38. Scale bar, $100 \mu \mathrm{m}$. (b-g) Cryosections of neocortical structures immunostained for various cortical markers at day 38, (b) BFI/PAX6 and Ki67/PAX6, (c) PAX6/Ncad, CDI33/PAX6, and g-tubulin/PAX6, (d) PH3/DAPI, (e) TUJI/PAX6, (f) DAPI/TBRI, and (g) REELIN/TBRI. Scale bar, $50 \mu \mathrm{m}$ for (b-f). Scale bar, $25 \mu \mathrm{m}$ for (g). (h) Phase-contrast image of a floating neocortical organoid derived from hPSCs at day 66. Scale bar, $100 \mu \mathrm{m}$. (i-k, r) Cryosections of neocortical structures immunostained for various cortical markers at day 66, (i) TUJI/DAPI, (j) PAX6/NEUN, (k) BFI/PAX6, and (r) DAPI/GFAP. BrdU incorporation (I h; I $0 \mu \mathrm{M})$ in the PAX6 ${ }^{+}$neuroepithelium at day 66 is shown in (I). (m-q) Immunofluorescence staining for (m) TUJI/Glutamate, (n) TUJINGLUTI, (o) TUJINGLUT2, (p) TUJI/GABA, and (q) TUJI/NGAT in the neocortical organoids at day 66. Scale bar, $50 \mu \mathrm{m}$ for (i-r). Cell line: H9. 
were also observed under voltage clamp conditions (Figure $3 d-f)$.

In contrast to the dual SMAD- and FGF-inhibition protocol that induced formation of self-organized neocortical organoids from larger rosettes (Figure 1d), most organoids that derived from smaller rosettes, without inhibitors or with only dual SMAD inhibitors (Figure 1d), did not recapitulate structural aspects of neocortical tissues (Figure $2 \mathrm{a}$ and $\mathrm{h}$ for blockade of dual SMAD and FGF signaling; Supplementary Figure S3a for no inhibitors; and Supplementary Figure S3b). Organoids derived from small rosettes without inhibitors did not display apicobasal arrangements of distinct neocortical zones such as $\mathrm{PAX}^{+}$neuroepithelia and $\mathrm{TUJ1}^{+}$neuronal layer formation (Supplementary Figure S3c and d; compare with Figure 2e, $i$ and $j$ ), suggesting that inhibition of dual SMAD and FGF signaling is essential for this selforganization.

Taken together, these data indicate that blockade of dual SMAD and FGF signaling at the start of differentiation induced formation of self-organized neocortical organoids, mimicking in vivo neocortical cytoarchitectural organization, and resulted in the establishment of functional neocortical neurons. We have successfully used this protocol on six different hPSC lines, including hESC lines H1, H9, H14, and $\mathrm{CT} 2$, and hiPSC lines $\mathrm{NIH}-\mathrm{i} 5$ and $\mathrm{NIH}-\mathrm{i} 7$, and obtained similar results (Supplementary Figure S4a-d).

\section{CYP3A5 and CYP3A43 Expression in Neocortical Organoids}

Our previous findings indicate that CYP450 inhibitors could reverse cocaine-induced impairment of neocortical development in both rat and 2D hPSC-based neocortical models (Lee et al, 2008; Kindberg et al, 2014). The specific isoforms of CYP450 that are responsible for the adverse effects of cocaine in the developing human neocortex are, however, unknown.

Because of the temporally sensitive effects of cocaine in rats, with neocortical abnormalities observed only when cocaine is administered during the most active period of neural progenitor proliferation (Lee et al, 2011), we examined the expression of CYP3A4, CYP3A5, CYP3A7, and $C Y P 3 A 43$ at the undifferentiated hPSC stage and in the neocortical organoids at day 44, during which time the neuroepithelium is rapidly proliferating (Figure 4a). CYP3A5 was expressed predominantly at the neocortical organoid stage, whereas CYP3A43 was expressed during both the undifferentiated and organoid stages (Figure 4a). Expression of CYP3A4 and CYP3A7 was not detected at either stage (Figure 4a). These data pinpoint CYP3A5 and CYP3A43 as prospective candidates for the metabolism-mediated developmental effects of cocaine.

\section{CYP3A5 Is Required for Cocaine-Induced Alterations in Neocortical Organoid Development}

We next examined the effects of cocaine on neocortical development using our 3D neocortical organoid model. In the fetal rat neocortex, cocaine concentrations were found to decrease rapidly to negligible levels $1 \mathrm{~h}$ after cocaine administration (Lee et al, 2008). Thus, in order to expose the neocortical organoids to an amount of cocaine that reflects in vivo exposure, the organoids were incubated with $3 \mu \mathrm{M}$ cocaine for $1 \mathrm{~h}$, followed by changing the culture medium. Organoids were exposed to cocaine every second day from days 32 to 44 . This represents the most active period of proliferation of the neuroepithelium in our organoids (Figure 2b). We measured ROS generation in neocortical organoids on day 44 and observed cocaineinduced ROS accumulation (Figure 4b). Cocaine also inhibited proliferation of $\mathrm{PAX}^{+}{ }^{+} \mathrm{NE}$ cells in neocortical organoids at day 45 (Figure $4 \mathrm{~d}$ and Supplementary Figure S5a). In the present model, postmitotic neurons migrate toward the periphery of the NE. Neurogenesis was examined using BrdU pulse-chase experiments ( $1 \mathrm{~h}$ BrdU pulse, $24 \mathrm{~h}$ chase) from days 51 to 52 , showing that cocaine increased the migration of BrdU-labeled TUJ1 neurons outwards to the periphery of the NE area (Figure $4 \mathrm{f}$ ). The percentage of $\mathrm{BrdU}^{+}$cells that expressed TUJ1 was increased by cocaine as compared with control (Figure $4 \mathrm{f}$ and Supplementary Figure S5b). These data confirm our previous findings, using a $2 \mathrm{D}$ neocortical model, that cocaine exposure led to premature neuronal differentiation (Kindberg et al, 2014). Finally, on day 66, at the end of differentiation, cocaine significantly inhibited development of the $\mathrm{TUJ}^{+}$area (Figure $4 \mathrm{~h}$ and Supplementary Figure S5c). These data demonstrate that $3 \mathrm{D}$ neocortical organoids can recapitulate cocaine-induced developmental abnormalities of the human neocortex.

To explore the role of CYP3A5 and CYP3A43 in these effects, we employed lentiviral vectors to deliver shRNA to silence either CYP3A5 or CYP3A43 using the H9 hESC line (Supplementary Figure S6). Knockdown of CYP3A5, but not CYP3A43, reversed cocaine-induced ROS generation (Figure 4c), proliferation inhibition (Figure 4e), and premature neuronal differentiation (Figure $4 \mathrm{~g}$ ). The overall inhibition of neural tissue development by cocaine was reversed only by CYP3A5 knockdown (Figure 4i). Moreover, knockdown of CYP3A5 or CYP3A43 itself did not interfere with neocortical development (Supplementary Figure S7a-d). These data indicate that CYP3A5, predominantly expressed during the beginning phase of neocorticogenesis, plays a dominant role in the adverse effects of cocaine in developing neocortical organoids.

Both CYP3A5 and CYP3A43 were expressed in dorsal forebrain cortical progenitors at day 16 at levels similar to those seen during early neocorticogenesis at day 44 (Supplementary Figure S8). Expression of CYP3A5 rapidly decreased after day 44 when neuronal differentiation gradually became dominant, whereas expression of CYP3A43 was maintained at a similar level until the end of differentiation (Supplementary Figure S8). These data lend further support to the hypothesis that the neocortex is most vulnerable to cocaine during early neocorticogenesis. Cocaine primarily alters neocortical development if administered when the neocortical organoids are still composed mainly of proliferative NE cells with high levels of CYP3A5 expression.

\section{DISCUSSION}

Recently, several hPSC-based 3D in vitro models of cerebral cortical development have been developed, and some 


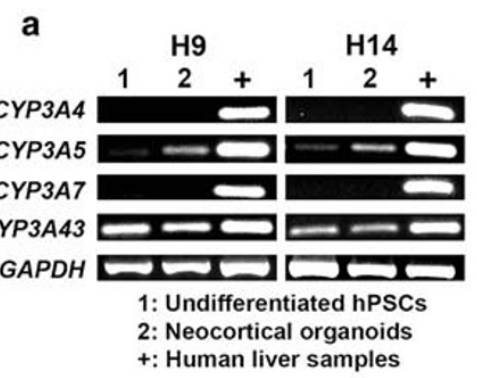

b
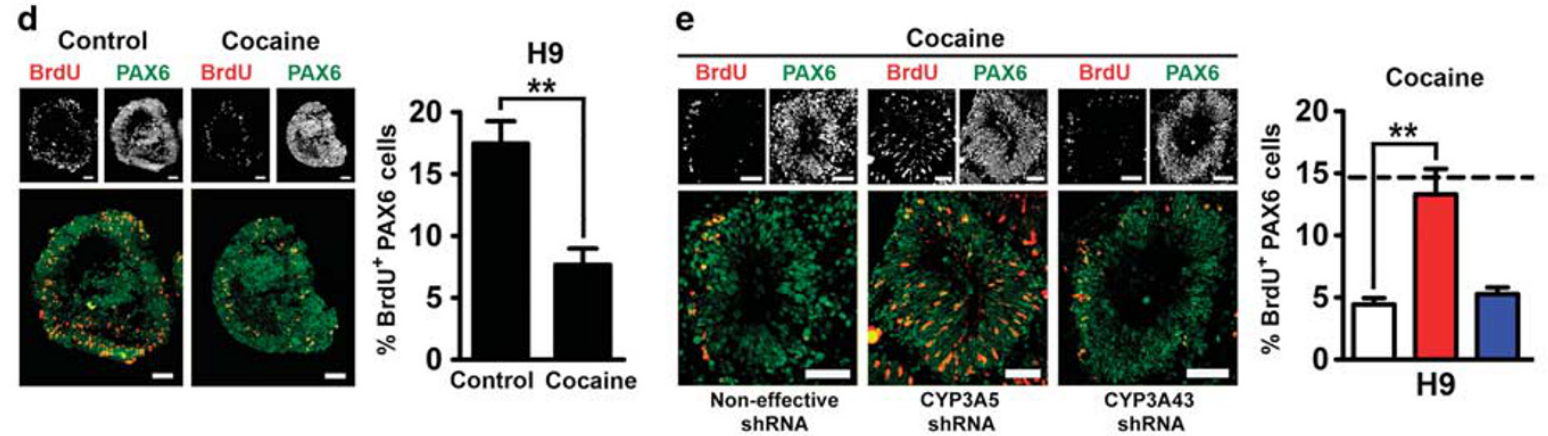

f
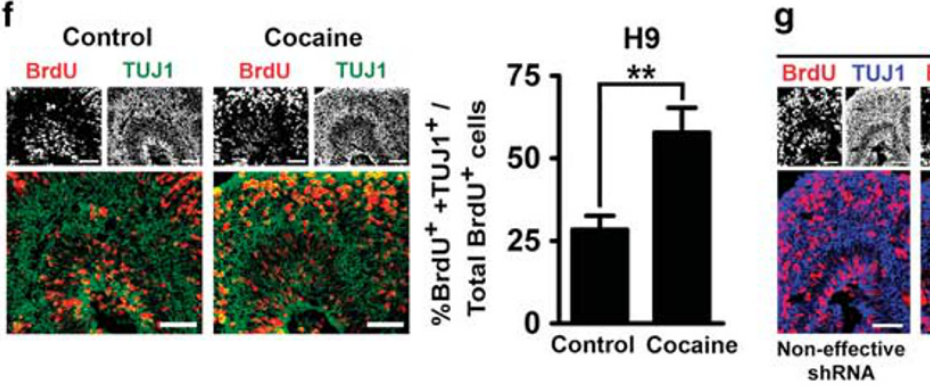

Cocaine

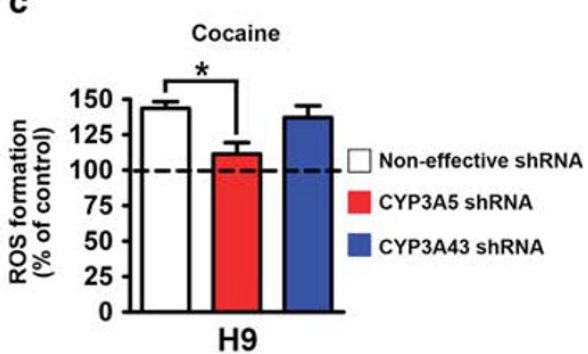

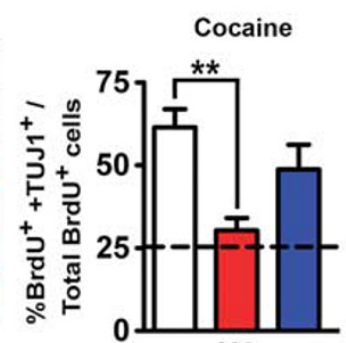

$\mathrm{H} 9$ h

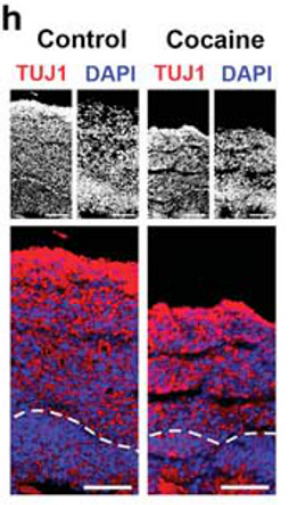

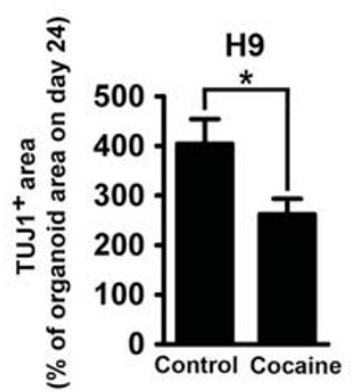

i

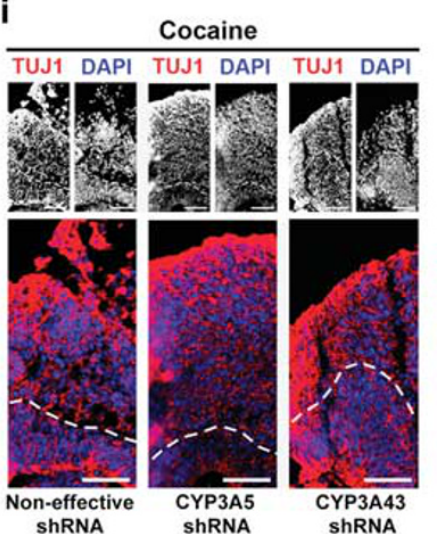

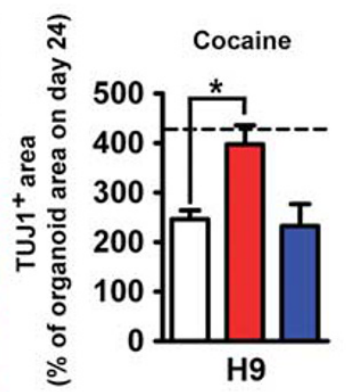

Figure 4 Neurodevelopmental effects of cocaine mediated by CYP3A5. (a) RT-PCR analysis showing expression of CYP3A family genes at different stages of neocortical organoid differentiation. 'Neocortical organoids' indicates day 44 of differentiation. (b) ROS formation in the neocortical organoids in the presence or absence of cocaine. Neocortical organoids were treated with $3 \mu \mathrm{M}$ cocaine for I h every other day from day 32 to day 44 , and endogenous ROS was measured at day 44; $n=6$. (c) Effects of CYP3A5 or CYP3A43 knockdown on cocaine-induced ROS generation in H9 (dashed line, in the absence of cocaine); $n=6$. (d) Proliferation of PAX $6^{+} \mathrm{NE}$ cells of neocortical organoids in the presence or absence of cocaine in $\mathrm{H}$ 9. Proliferation was examined at day 45 with I h BrdU $(10 \mu M)$ incorporation. Corresponding percentages of BrdU-labeled PAX $6^{+}$cells are shown at right. Scale bar, $50 \mu m ; n=5$. (e) Effects of CYP3A5 or CYP3A43 knockdown on cocaine-induced inhibition of proliferation of PAX $6^{+} \mathrm{NE}$ cells in $\mathrm{H} 9$ (dashed line, in the absence of cocaine). Scale bar, $50 \mu \mathrm{m} ; n=7$. ( $f$ Neuronal differentiation in neocortical organoids in the presence or absence of cocaine in $\mathrm{H} 9$. Neuronal differentiation was analyzed $24 \mathrm{~h}$ after I h BrdU (IO $\mu \mathrm{M})$ treatment from day $5 \mathrm{I}$ to day 52. Corresponding percentages of $\mathrm{BrdU}^{+}$cells exhibiting neuronal identity $\left(\mathrm{TU} \mathrm{I}^{+}\right)$are shown at right. Scale bar, $50 \mu \mathrm{m} ; n=5$. (g) Effects of CYP3A5 or CYP3A43 knockdown on cocaine-induced premature neuronal differentiation in H9 (dashed line, in the absence of cocaine). Scale bar, $50 \mu \mathrm{m} ; n=7$. (h) Neural tissue development in the presence or absence of cocaine in $\mathrm{H9}$. TUJ ${ }^{+}$areas in the neocortical organoids were analyzed at day 66. Corresponding TUJI+ areas expressed as percentages of total organoid area on day 24 are shown at right. Scale bar, $50 \mu \mathrm{m} ; n=6$. (i) Effects of CYP3A5 or CYP3A43 knockdown on cocaine-induced inhibition of neural tissue development in H9 (dashed line, in the absence of cocaine). Scale bar, $50 \mu m ; n=6$. Data are shown as means \pm SEM. Unpaired two-tailed Student's t-test for H9 and HI4 in (b) and for (d, f, h). One-way ANOVA followed by Tukey's compromise post hoc test for (c, g, i). Dunn's multiple comparisons test for (e). ${ }^{*} P<0.05$ and ${ }^{*} * P<0.0$ l. 
of them have been used to recapitulate complex disorders of brain development, such as microcephaly (Lancaster et al, 2013; Paşca et al, 2015). However, using gelatinous protein mixtures to serve as scaffolding for the developing brain organoids could limit their exposure to test compounds, as well as introducing undefined components of animal origin. Moreover, generation of unpredictable numbers of $\mathrm{NE}$ rosettes in cortical organoids could interrupt the enlargement of the neocortical area between the borders of adjacent neocortical structures that causes difficulty in obtaining reproducible measurements. Therefore, these methods are not suitable for certain pharmacological applications that require quantification. Here, we developed a 3D hPSC-based neocortical organoid model in which a single neocortical structure self-organizes without the support of a gelatinous matrix. These organoids retain several essential features of human neocortical development, thus allowing us to examine the mechanisms involved in the adverse neurodevelopmental effects of psychostimulants, in this case cocaine.

Using our neocortical organoid model, we found that CYP3A5, predominantly expressed at the early stage of neocorticogenesis, plays a critical role in the developmental effects of cocaine. Human CYP3A is important for the oxidative metabolism of many drugs including psychostimulants. Moreover, its location in the brain suggests a potential role of CYP3A in pharmacological modulation of drug-related brain activity. Among the CYP3A family, CYP3A4, CYP3A5, and CYP3A43 have been detected in different regions of the adult human brain, including cortex, hippocampus, basal ganglia, midbrain, and cerebellum (Booth Depaz et al, 2013; McFadyen et al, 1998; Agarwal et al, 2008). However, the expression pattern of CYP3A in the human embryonic brain is not clear. We found that only CYP3A5 and CYP3A43 are expressed in the neocortical organoids (Figure 4a). Furthermore, knockdown of CYP3A5, but not CYP3A43, reversed cocaine-induced alterations in neocortical development (Figure 4c, e, g and i), suggesting that CYP3A5 as a biological driver for cocaine-induced brain developmental deficits.

Although the primary recognized function of CYP3A5 involves metabolism of drugs and various compounds, the present study does not demonstrate that metabolism of cocaine per se is responsible for the effects of cocaine on development of cortical organoids. We cannot exclude the possibility that other unknown mechanisms involving CYP3A5 are involved. In addition, as cocaine has been shown to affect adult neurogenesis in the rat subgranular zone of the hippocampus (Noonan et al, 2008), and CYP3A5 has been shown to be expressed in the human hippocampus (Booth Depaz et al, 2013; McFadyen et al, 1998), it would be interesting to determine whether CYP3A5 is involved in cocaine-induced disruption of neurogenesis in this brain region.

In conclusion, the primary advantages of our hPSC-based neocortical organoid model are, first, that it comprises a 3D model that retains only one neocortex-like structure organized around a single ventricle-like structure, and, second, that no animal-derived gelatinous matrix is used. Studies performed using this in vitro $3 \mathrm{D}$ model indicate that at least some effects of cocaine on neocortical development are mediated by CYP3A5. As CYP3A isozymes have been shown to metabolize $\sim 50 \%$ of all currently used therapeutic drugs, it may also be critical for neocortical development disorders observed with prenatal exposure to drugs other than cocaine. A detailed examination of chemical compounds and drugs metabolized by CYP3A on brain development using our 3D neocortical organoid model could lead to identification of adverse effects of pharmaceuticals in humans, and development of improved therapeutics for specific drug exposure. The hPSC-based human tissue models are widely utilized for drug safety screening, especially using cardiomyocytes and hepatocytes (Grimm et al, 2015; Sengupta et al, 2014); however, the present study suggests that hPSC-based models can also be used to obtain information about molecular mechanisms and potential therapeutical targets in drug-induced disease. A previous study by our group (Zeng et al, 2006) demonstrated that hESC-based models could be employed for studies of toxicity of human dopaminergic systems; the present study and our previous report using a 2D model (Kindberg et al, 2014) expands that concept to include the neocortex.

Stem cell technology using patient-derived hiPSCs also enables the development of human in vitro model systems that capture the inherent pathologies based on the genetic background of the source material (Lee et al, 2015c; Aigner et al, 2014; Brennand et al, 2011, 2012). Some neurodevelopmental disorders such as autism spectrum disorder (ASD) and psychiatric illnesses such as schizophrenia, with dysfunction in the prefrontal cortex (Courchesne et al, 2011; Stoner et al, 2014; Anticevic et al, 2015), only occur in humans. Animal models can recapitulate only selected aspects of these complex disorders (Ruhela et al, 2015; Ellenbroek and Cools, 2000). Our 3D neocortical organoid model offers distinct advantages for the study of these inherited neurodevelopmental and psychiatric disorders. This system might be profitably used to examine the underlying genetic factors in neurological disorders using hiPSC-derived 3D neocortical organoids.

\section{FUNDING AND DISCLOSURE}

The authors declare no conflict of interest.

\section{ACKNOWLEDGMENTS}

A patent application has been filed for the $3 \mathrm{D}$ model (WJ Freed and C-T Lee). This work was supported by the Intramural Research Program of the National Institute on Drug Abuse.

\section{REFERENCES}

Agarwal V, Kommaddi RP, Valli K, Ryder D, Hyde TM, Kleinman JE et al (2008). Drug metabolism in human brain: high levels of cytochrome P4503A43 in brain and metabolism of anti-anxiety drug alprazolam to its active metabolite. PLoS One 3: e2337.

Aigner S, Heckel T, Zhang JD, Andreae LC, Jagasia R (2014). Human pluripotent stem cell models of autism spectrum disorder: emerging frontiers, opportunities, and challenges towards neuronal networks in a dish. Psychopharmacology (Berl) 231: 1089-1104. 
Anticevic A, Hu X, Xiao Y, Hu J, Li F, Bi F et al (2015). Early-course unmedicated schizophrenia patients exhibit elevated prefrontal connectivity associated with longitudinal change. J Neurosci 35: 267-286.

Bandstra ES, Morrow CE, Accornero VH, Mansoor E, Xue L, Anthony JC (2011). Estimated effects of in utero cocaine exposure on language development through early adolescence. Neurotoxicol Teratol 33: 25-35.

Bandstra ES, Vogel AL, Morrow CE, Xue L, Anthony JC (2004). Severity of prenatal cocaine exposure and child language functioning through age seven years: a longitudinal latent growth curve analysis. Subst Use Misuse 39: 25-59.

Booth Depaz IM, Toselli F, Wilce PA, Gillam EM (2013). Differential expression of human cytochrome P450 enzymes from the CYP3A subfamily in the brains of alcoholic subjects and drug-free controls. Drug Metab Dispos 41: 1187-1194.

Brennand KJ, Simone A, Jou J, Gelboin-Burkhart C, Tran N, Sangar S et al (2011). Modelling schizophrenia using human induced pluripotent stem cells. Nature 473: 221-225.

Brennand KJ, Simone A, Tran N, Gage FH (2012). Modeling psychiatric disorders at the cellular and network levels. Mol Psychiatry 17: 1239-1253.

Cameron RS, Rakic P (1991). Glial cell lineage in the cerebral cortex: a review and synthesis. Glia 4: 124-137.

Chambers SM, Fasano CA, Papapetrou EP, Tomishima M, Sadelain M, Studer L (2009). Highly efficient neural conversion of human ES and iPS cells by dual inhibition of SMAD signaling. Nat Biotechnol 27: 275-280.

Chung K, Wallace J, Kim SY, Kalyanasundaram S, Andalman AS, Davidson TJ et al (2013). Structural and molecular interrogation of intact biological systems. Nature 497: 332-337.

Courchesne E, Mouton PR, Calhoun ME, Semendeferi K, AhrensBarbeau C, Hallet MJ et al (2011). Neuron number and size in prefrontal cortex of children with autism. JAMA 306: 2001-2010.

Dehay C, Kennedy H (2007). Cell-cycle control and cortical development. Nat Rev Neurosci 8: 438-450.

Ellenbroek BA, Cools AR (2000). Animal models for the negative symptoms of schizophrenia. Behav Pharmacol 11: 223-233.

Greber B, Coulon P, Zhang M, Moritz S, Frank S, Müller-Molina AJ et al (2011). FGF signalling inhibits neural induction in human embryonic stem cells. EMBO J 30: 4874-4884.

Grimm FA, Iwata Y, Sirenko O, Bittner M, Rusyn I (2015). Highcontent assay multiplexing for toxicity screening in induced pluripotent stem cell-derived cardiomyocytes and hepatocytes. Assay Drug Dev Technol 13: 529-546.

Hasegawa H, Ashigaki S, Takamatsu M, Suzuki-Migishima R, Ohbayashi N, Itoh $\mathrm{N}$ et al (2004). Laminar patterning in the developing neocortex by temporally coordinated fibroblast growth factor signaling. J Neurosci 24: 8711-8719.

Hill RS, Walsh CA (2005). Molecular insights into human brain evolution. Nature 437: 64-67.

Kindberg AA, Bendriem RM, Spivak CE, Chen J, Handreck A, Lupica CR et al (2014). An in vitro model of human neocortical development using pluripotent stem cells: cocaineinduced cytoarchitectural alterations. Dis Model Mech 7: 1397-1405.

Lancaster MA, Renner M, Martin CA, Wenzel D, Bicknell LS, Hurles ME et al (2013). Cerebral organoids model human brain development and microcephaly. Nature 501: 373-379.

Lee CT, Bendriem RM, Freed WJ (2015a). A new technique for modeling neuronal connectivity using human pluripotent stem cells. Restor Neurol Neurosci 33: 347-356.

Lee CT, Bendriem RM, Kindberg AA, Worden LT, Williams MP, Drgon $\mathrm{T}$ et al (2015b). Functional consequences of 17q21.31/ WNT3-WNT9B amplification in hPSCs with respect to neural differentiation. Cell Rep 10: 616-632.
Lee CT, Chen J, Hayashi T, Tsai SY, Sanchez JF, Errico SL et al (2008). A mechanism for the inhibition of neural progenitor cell proliferation by cocaine. PLoS Med 5: e117.

Lee CT, Chen J, Worden LT, Freed WJ (2011). Cocaine causes deficits in radial migration and alters the distribution of glutamate and GABA neurons in the developing rat cerebral cortex. Synapse 65: 21-34.

Lee CT, Freed WJ, Mash DC (2015c). CNVs in neurodevelopmental disorders. Oncotarget 6: 18238-18239.

Levison SW, Goldman JE (1993). Both oligodendrocytes and astrocytes develop from progenitors in the subventricular zone of postnatal rat forebrain. Neuron 10: 201-212.

Lidow MS, Bozian D, Song ZM (2001). Cocaine affects cerebral neocortical cytoarchitecture in primates only if administered during neocortical neuronogenesis. Brain Res Dev Brain Res 128: 45-52.

Lidow MS, Song ZM (2001). Primates exposed to cocaine in utero display reduced density and number of cerebral cortical neurons. J Comp Neurol 435: 263-275.

Mallon BS, Chenoweth JG, Johnson KR, Hamilton RS, Tesar PJ, Yavatkar AS et al (2013). StemCellDB: the human pluripotent stem cell database at the National Institutes of Health. Stem Cell Res 10: 57-66.

Martignoni M, Groothuis GM, de Kanter R (2006). Species differences between mouse, rat, dog, monkey and human CYPmediated drug metabolism, inhibition and induction. Expert Opin Drug Metab Toxicol 2: 875-894.

McFadyen MC, Melvin WT, Murray GI (1998). Regional distribution of individual forms of cytochrome P450 mRNA in normal adult human brain. Biochem Pharmacol 55: 825-830.

Noonan MA, Choi KH, Self DW, Eisch AJ (2008). Withdrawal from cocaine self- administration normalizes deficits in proliferation and enhances maturity of adult-generated hippocampal neurons. J Neurosci 28: 2516-2526.

Paşca AM, Sloan SA, Clarke LE, Tian Y, Makinson CD, Huber N et al (2015). Functional cortical neurons and astrocytes from human pluripotent stem cells in 3D culture. Nat Methods 12: 671-678.

Raballo R, Rhee J, Lyn-Cook R, Leckman JF, Schwartz ML, Vaccarino FM (2000). Basic fibroblast growth factor (Fgf2) is necessary for cell proliferation and neurogenesis in the developing cerebral cortex. J Neurosci 20: 5012-5023.

Rakic P (2009). Evolution of the neocortex: a perspective from developmental biology. Nat Rev Neurosci 10: 724-735.

Rando K, Chaplin TM, Potenza MN, Mayes L, Sinha R (2013). Prenatal cocaine exposure and gray matter volume in adolescent boys and girls: relationship to substance use initiation. Biol Psychiatry 74: 482-489.

Ruhela RK, Prakash A, Medhi B (2015). An urgent need for experimental animal model of autism in drug development. Ann Neurosci 22: 44-49.

Sarnat HB, Nochlin D, Born DE (1998). Neuronal nuclear antigen (NeuN): a marker of neuronal maturation in early human fetal nervous system. Brain Dev 20: 88-94.

Sengupta S, Johnson BP, Swanson SA, Stewart R, Bradfield CA, Thomson JA (2014). Aggregate culture of human embryonic stem cell-derived hepatocytes in suspension are an improved in vitro model for drug metabolism and toxicity testing. Toxicol Sci 140: 236-245.

Singer LT, Arendt R, Minnes S, Farkas K, Salvator A, Kirchner HL et al (2002). Cognitive and motor outcomes of cocaine-exposed infants. JAMA 287: 1952-1960.

Singer LT, Nelson S, Short E, Min MO, Lewis B, Russ S et al (2008). Prenatal cocaine exposure: drug and environmental effects at 9 years. J Pediatr 153: 105-111.

Stoner R, Chow ML, Boyle MP, Sunkin SM, Mouton PR, Roy S et al (2014). Patches of disorganization in the neocortex of children with autism. N Engl J Med 370: 1209-1219. 
Supèr H, Uylings HB (2001). The early differentiation of the neocortex: a hypothesis on neocortical evolution. Cereb Cortex 11: 1101-1109.

Takahashi T, Nowakowski RS, Caviness VS Jr (1993). Cell cycle parameters and patterns of nuclear movement in the neocortical proliferative zone of the fetal mouse. J Neurosci 13: 820-833.
Valente MJ, Carvalho F, Bastos Md, de Pinho PG, Carvalho M (2012). Contribution of oxidative metabolism to cocaine-induced liver and kidney damage. Curr Med Chem 19: 5601-5606.

Zeng X, Chen J, Deng X, Liu Y, Rao MS, Cadet JL et al (2006). An in vitro model of human dopaminergic neurons derived from embryonic stem cells: MPP+ toxicity and GDNF neuroprotection. Neuropsychopharmacology 31: 2708-2715.

Supplementary Information accompanies the paper on the Neuropsychopharmacology website (http://www.nature.com/npp) 